\title{
Preface
}

\section{Uniportal video-assisted thoracic surgery}

Even though the development of thoracoscopic procedures has a history of over a hundred years, it is only in the last two decades that interest in minimally invasive thoracic surgery has been renewed by two key technological improvements. These are the development of endoscopic cameras offering a panoramic view and the availability of new endoscopic instruments such as mechanical staplers. As a result of these advances, video-assisted thoracic surgery (VATS) was born. In 1992, there was a revolution in thoracic surgery when the first anatomic lung resection was performed by VATS through small incisions and with no rib spreading.

In the following years, the progress of VATS was slow and accompanied by constant criticism until studies showing clear benefits of VATS over open surgery started to be published. From that point on, the technique spread throughout the world and variations of the technique regarding the number and placement of incisions, surgical approaches and new surgical instruments started to emerge. The information available on the Internet, live surgical events and experimental courses were important factors facilitating the rapid learning curve for minimally invasive surgery during the last decade. While initially slow to catch on, the traditional multi-port approach has evolved into a single-port (uniportal) approach that mimics open surgical vantage points while utilizing a non-rib-spreading, non-thoracotomy micro-incision. The early development of uniportal VATS was focused on minor procedures until the second phase of uniportal VATS started in 2010 with application of the technique to major pulmonary resections. In a period of only five to six years, experts have been able to apply the uniportal VATS technique to more complex procedures such as bronchial sleeve and carinal resections as well as vascular reconstructions. In contrast, non-intubated thoracic surgical techniques, described since the early history of thoracic surgery, peaked in the decades before the invention of the double-lumen endotracheal tube and have failed to gain widespread acceptance following their re-emergence over a decade ago thanks to improvements in video-assisted thoracoscopic techniques. In addition, experienced uniportal VATS surgeons are able to explore new and even less invasive approaches. Recently, the aim to avoid intercostal nerve damage by the transthoracic incision has led to the use of the technique through a single subxiphoid incision.

It's interesting to note that from the first thoracoscopic procedure performed more than a century ago, the biggest developments have taken place in the last five years. Several factors are responsible for this. The Internet has provided an invaluable platform to share knowledge. The development of specialized websites to which videos can be uploaded and scientific journals that include videos have contributed enormously to the dissemination and learning of minimally invasive techniques, especially uniportal VATS. The development of modern, narrowed and angulated endostaplers and other specialized surgical instruments have also been key factors contributing to the growing interest in uniportal video-assisted surgery in the last few years. At the same time, advances in the technology of ultra-high definition cameras, 3D technology and precise robotic systems have contributed to the progressive adoption of minimally invasive surgery throughout the globe.

This special issue provides a summary of different aspects of uniportal VATS including several articles written by some of the best world experts in this technique. Areas covered range from the early experience of several groups to reports from the most experienced surgical teams, as well as emerging techniques such as uniportal non-intubated and subxiphoid approaches. Alongside medical and radiation oncology, which are advancing rapidly due to the development of more effective chemotherapeutic agents with less toxicity and novel radiation techniques such as stereotactic body radiation therapy (SBRT), the role of the surgeon is to offer the patient the best oncologic procedure with the least invasive approach possible.

During the last couple of years, rapid progress in instrument design and technology have triggered the development of narrower and more angulated endostaplers, sealing devices for vessels and other refined thoracoscopic instruments. We truly believe in the use of the least invasive techniques such as the uniportal approach combined with modern 'naked eye' 3D image systems, wireless cameras and improved robotic technology to perform major pulmonary resections. Further advancements in technology will facilitate the adoption of single-incision minimally invasive techniques worldwide in the upcoming years.

Diego Gonzalez-Rivas, MD, FECTS

(Email: diego.gonzalez.rivas@sergas.es.)

Director Uniportal VATS Training Program, Shangbai Pulmonary Hospital, Tongji University, Shanghai 200433, China

doi: 10.21037 /acs.2016.03.04

Conflicts of Interest: The author has no conflicts of interest to declare.

View this article at: http://dx.doi.org/10.21037/acs.2016.03.04 